\section{Articulação entre serviços públicos de saúde nos cuidados voltados à saúde mental infantojuvenil}

\author{
Articulation between child and adolescent \\ mental health services
}

\author{
1 Universidade de São Paulo, \\ São Paulo, Brasil. \\ Correspondência \\ P. S. S. Delfini \\ Universidade de São Paulo. \\ Av. Dr. Arnaldo 715, sala 341, \\ São Paulo, SP 02040-120, \\ Brasil. \\ patriciadelfini@usp.br
}

\section{Abstract}

The objective of this paper was to describe and analyze the articulation between children and adolescent mental health care interventions undertaken by teams working under the Family Health Strategy (FHS) and Psychosocial Care Centers for Children and Adolescents (CAPSI) for. In order to achieve these objectives, semi-structured interviews were conducted with five CAPSI and 13 FHS managers from five different regions of the city of São Paulo, Brazil. The 18 interviews were transcribed and analyzed froma hermeneutic perspective. It was found that interactions between the FHS and CAPSI occur mainly through referral of cases, matrix support or partnerships in cases concerning CAPSI. Obstacles, such as a lack of human resources, productivity goals and lack of training in mental health of FHS professionals were mentioned. The referral system and passing responsibility for mental health cases to specialized services and the hegemonic biomedical model and the fragmentation of care are common place in these services.

Mental Health; Mental Health Services; Child; Adolescent
Patricia Santos de Souza Delfini 1 Alberto Olavo Advincula Reis 1

\section{Introdução}

A atenção voltada à saúde mental de crianças e adolescentes e seu reconhecimento como uma questão de saúde pública integrante das ações do Sistema Unico de Saúde (SUS) é recente e tem sido considerada como um dos principais desafios da Reforma Psiquiátrica brasileira. O movimento da Reforma Sanitária, sedimentada com construção do SUS, e o reconhecimento da criança e do adolescente como sujeitos de direitos e responsabilidades pela promulgação do Estatuto da Criança e do Adolescente (ECA), de 1990, redefiniram a posição do Estado em relação à assistência e à elaboração de políticas públicas voltadas a tal população 1 .

Diretrizes ministeriais 2 sugerem que os cuidados em saúde mental infantojuvenil se desenvolvam em diversos serviços de saúde, como atenção básica - Unidades Básicas de Saúde (UBS) e Estratégia Saúde da Família (ESF), Centros de Atenção Psicossocial Infantojuvenil (CAPSI), ambulatórios e hospitais gerais que articulados a uma rede intersetorial têm como maior meta a inclusão social de seus usuários.

O CAPSI, serviço de atenção diária voltado a crianças e adolescentes com grave comprometimento psíquico 2 , constitui a principal estratégia vigente na atualidade do processo da Reforma Psiquiátrica Brasileira. Pesquisas atuais indicam que ele tem atendido especialmente crianças e adolescentes do sexo masculino com hipótese 
diagnóstica de trantorno de comportamento e emocional $3,4,5$.

Entretanto, a atenção básica tem sido considerada lugar privilegiado para a construção de uma nova lógica de cuidados em saúde mental, já que por sua proximidade com a comunidade, seus profissionais se deparam frequentemente com problemas de saúde mental e podem ser um recurso estratégico para o enfrentamento dessas questões. Segundo pesquisa do Ministério da Saúde, $56 \%$ das equipes de saúde da família referem realizar "alguma ação de saúde mental" em seu cotidiano ${ }^{6}$. Todavia, essas equipes nem sempre apresentam condições para darem conta das situações e necessitam de apoio.

Dessa forma, é importante que os CAPS e as equipes da rede básica trabalhem de maneira integrada para que ambas compartilhem a responsabilidade pelos casos e garantam maior resolutividade no manejo das situações que envolvam sofrimento psíquico 7 .

Apesar dessa importância, pesquisas indicam que diversas dificuldades são encontradas no dia a dia de trabalho dessas equipes. Existem muitos desafios a serem superados 8,9,10,11,12,13,14, destacando-se: falta de capacitação das equipes de saúde da família e daquelas dos novos serviços de saúde mental para um pensar e agir voltado à atenção psicossocial, falta de espaços de reflexão e análise sobre o trabalho e sobre a loucura, falta de entrosamento entre as equipes da ESF e os serviços especializados, escassez de serviços ou de profissionais de saúde mental e baixa cobertura da ESF em certas regiões. Tais carências geram, entre outras consequências, superlotação dos serviços, sentimento de sobrecarga por parte dos trabalhadores advindo do contato cotidiano com o sofrimento e isolamento dos serviços em si mesmos.

São escassos os estudos que levam em conta os cuidados voltados à população infantojuvenil e à articulação dos serviços de saúde para esse fim. O desenvolvimento de pesquisas com esse foco tem importância não só devido à contemporaneidade do tema, como também por poder contribuir para a melhoria da atenção em saúde mental oferecida pelos atuais serviços públicos brasileiros por meio de produção de conhecimento.

O presente estudo, resultante de pesquisa de mestrado, descreve e analisa as articulações que se realizam entre as equipes da ESF e de CAPSI da cidade de São Paulo, Barsil, tendo em vista as ações voltadas à atenção em saúde mental infantojuvenil. Objetiva contribuir para a reflexão e o debate sobre o tema e fornecer subsídios adequados para avaliação e elaboração de diretrizes que se integram às políticas públicas para a população infantojuvenil capazes de fomentar e contemplar, de modo concreto, as facilidades e dificuldades enfrentadas no trabalho prático.

\section{Método}

Pesquisa descritiva, de natureza qualitativa, baseada em uma perspectiva hermenêutica. Ao adotar esse referencial analítico, supera-se o reducionismo de se compreender a abordagem qualitativa como teoria de tratamento de dados 15. Tal como postulado por Habermas, a hermenêutica determina, antes de tudo, a autocompreensão dos dados, não sendo limitada ao determinismo das técnicas de tratamento. Nossa escolha técnico-metodológica por uma "entrevista semidiretiva" assegurou a possibilidade de diálogo entre entrevistador-entrevistado como substrato necessário à operação hermenêutica visando à produção de confluência do familiar com o inesperado. $\mathrm{O}$ dado assim construído foi passível, então, de ser objeto de explicação e interpretação 16 .

Foram sujeitos da pesquisa gerentes de CAPSI e de unidades com ESF, atuantes em cinco regiões distintas da cidade de São Paulo. As unidades foram selecionadas tendo em vista os dez CAPSI existentes na cidade e habilitados pela prefeitura no início do trabalho, em 2008. Os critérios para escolha das unidades foram: (1) que o tempo de existência do serviço fosse superior a dois anos; (2) que o público atendido fosse tanto de crianças quanto adolescentes; (3) que as unidades selecionadas pertencessem a diferentes regiões da cidade. Para eleger as UBS com equipes de Saúde da Família, foi considerada a distância física de cada uma em relação aos CAPSI, e foram eleitas três unidades por região, sendo as duas mais próximas da unidade e uma mais afastada, desde que dentro da área de cobertura do CAPSI.

A escolha pelos gerentes das unidades se deu por terem sido avaliados como as pessoas que reúnem maior amplitude de informações sobre os aspectos organizacionais envolvidos na articulação entre os distintos serviços. Assim, fizeram parte da pesquisa os gerentes de 5 CAPSI e de 13 unidades da ESF, totalizando 18 entrevistados. Houve recusa de duas pessoas em participar da pesquisa.

As entrevistas realizadas abordaram: (1) descrição da articulação entre as equipes de ambos os serviços nas ações voltadas à saúde mental infantojuvenil; (2) impressões sobre a articulação existente; (3) facilitadores e obstáculos encontrados para esse fim.

Posteriormente à sua execução, as entrevistas gravadas foram transcritas e lidas repetidas ve- 
zes. O material foi submetido a técnicas de análise de conteúdo, entendida como "um conjunto de técnicas de análise de comunicação visando obter, por procedimentos sistemáticos e objetivos da descrição de conteúdos de mensagens, indicadores que permitam a inferência de conhecimento relativo às condições de produção/recepção destas mensagens" 17 (p. 42). Dentre as diversas modalidades de análise de conteúdo, optamos pela análise temática que propõe a descoberta de núcleos de sentido que fazem parte de uma comunicação cuja presença ou frequência possam significar algo para o objetivo analítico visado 15 . Nossa análise se dividiu em três etapas 15: préanálise, exploração do material e tratamento dos resultados.

Sendo assim, procedemos a esses passos, decompusemos o material colhido em núcleos de sentido, e aqueles que tiveram maior significância nas entrevistas em termos de repetição e frequência dentro da temática que objetivamos - articulação entre CAPSI e ESF no que se refere à saúde mental de crianças e adolescentes - serão apresentados neste estudo. São eles: encaminhamento, matriciamento e parceria para casos de CAPSI.

Os resultados encontrados foram analisados e discutidos tendo em vista aproximações e distanciamentos da prática cotidiana com conceitos norteadores do campo da saúde mental coletiva na perspectiva da atenção psicossocial ${ }^{18}$, tais como território, rede de cuidado e corresponsabilidade 19 .

Esta pesquisa cumpre as exigências éticas descritas na Resolução $n^{\circ}$. 196/1996, do Conselho Nacional de Saúde, que dispõe sobre as diretrizes e normas de pesquisas envolvendo seres humanos e foi aprovada no Comitê de Ética em Pesquisa da Faculdade de Saúde Pública, Universidade de São Paulo (protocolo no ${ }^{\circ}$ 1943) e da Secretaria Municipal de Saúde de São Paulo (parecer no. 79/09). O sigilo e a participação voluntária dos sujeitos da pesquisa foram garantidos mediante Termo de Consentimento Livre e Esclarecido.

\section{Análise e discussão dos resultados}

\section{Encaminhamento}

O encaminhamento se mostra como a principal forma de contato entre as equipes de ESF e dos CAPSI pesquisados. São atribuídos, entretanto, sentidos distintos para o termo, variando desde um encaminhamento indiscriminado, no qual encaminhar é sinônimo de se desresponsabilizar até um encaminhamento implicado, no qual se pede ajuda/apoio para situações que exigem al- guma especificidade e, em alguns casos, se compartilha o cuidado.

\section{- Encaminhar como passar a responsabilidade}

Nesse caso, tanto as equipes de ESF quanto de CAPSI que encaminham, "reencaminham" ou "devolvem o paciente" o fazem por meio telefônico, envio direto do usuário/família para a outra unidade, ou utilizando a central de regulação, sistema eletrônico do município em que se encontram vagas para especialidades.

Quando há algum contato entre profissionais das equipes, ele se dá apenas para verificar a disponibilidade de vaga no CAPSI e agendar uma triagem dos pacientes. Nas situações em que o encaminhamento é feito sem que haja contato entre as equipes, o usuário é enviado de um serviço ao outro à procura de cuidados, terminando, muitas vezes, sem acolhimento algum para seu sofrimento. Há, ainda, locais onde se usam centrais de regulação ou distribuição de "senhas", que são divididas entre as unidades da região, sem que exista qualquer relação com a singularidade ou necessidade do caso.

“(...) a gente só recebe, por exemplo, as senhas, né, têm... ah, eu tenho direito a 4 senhas no mês, 3 senhas, aí vai depender do número disponibilizado. Então tem uma fila de espera e aí a gente encaminha" (gerente ESF).

Percebemos, por falas como essa, que não há compartilhamento do projeto terapêutico, mas passagem de casos que são avaliados e pensados por cada equipe de forma isolada. Mesmo havendo um contato entre os profissionais, ele ocorre para agendamento da triagem do usuário, o qual é submetido a uma avaliação que definirá seu local de atendimento, que tende a ocorrer dentro de um ou de outro serviço. Esses intermináveis encaminhamentos evidenciam desarticulação das ações bem como uma relação fragmentada entre equipamentos, o que inviabiliza um atendimento integral e transdisciplinar 20.

“(...) é difícil a gente conseguir uma vaga ou quando você encaminha o paciente, ele retorna aqui pra gente. Então tinha que ter aquilo, né, se for encaminhado, mesmo que eles achem que não é o caso, entrassem em contato com a gente, conversasse, não mandasse o paciente vir. Aí ele vai, parece um ping-pong" (gerente ESF).

Essa forma de operar leva à fragmentação do cuidado e à descontinuidade do projeto terapêutico 19 . Ainda, perde-se a potência do trabalho conjunto e a ampliação das possibilidades de cuidado do usuário que não faz uso de um sistema integrado de saúde, mas de equipamentos isolados que tratam de problemas pontuais, o 
que aponta para um entendimento de que pessoas em sofrimento psíquico devam ser tratadas por especialistas de um serviço específico, em geral, o CAPS.

Cabe ressaltar que a existência de filas de espera fere o princípio do acolhimento universal, proposto pelo Ministério da Saúde que diz que todos os serviços públicos de saúde mental devem estar abertos a todos que cheguem, independentemente de sua capacidade de absorção ${ }^{2}$. Vemos aí uma lacuna entre a política preconizada e a prática dos serviços.

Adicionalmente, notamos que os processos de trabalho ocorrem com base num modelo de saúde tradicional, no qual o sujeito é visto como partes desarticuladas; não se valoriza o vínculo usuário-equipe, tampouco a integralidade do cuidado, características fundamentais para a atenção psicossocial e territorial. A fragmentação do cuidado decorre, em grande parte, do aumento crescente das especialidades e da forma como se organizam os processos de trabalho 19. Ao se trabalhar desse modo, a possibilidade de realização de uma clínica ampliada - entendida como aquela cujo olhar é voltado para o sujeito e toda sua complexidade, em contraposição à clínica voltada apenas para a doença e seus sintomas fica comprometida.

A "lógica do encaminhamento" - na qual os usuários do sistema são direcionados a outros serviços para procurar de atendimento, e os trabalhadores fazem uso de instâncias burocráticas e hierarquizadas para se articularem uns com os outros - leva a uma diluição, e não a um compartilhamento, da responsabilidade sobre os casos 19.

Salientamos que a ESF tem como foco o cuidado integral e longitudinal de sua população. É uma equipe de referência que acompanha a saúde geral das famílias, mesmo quando um usuário necessita de alguma especificidade. Importante dizer que algumas entrevistas indicam uma crítica ao modelo fragmentado e relatam que o trabalho conjunto tem, progressivamente, ajudado a superar esse modelo.

"Foi difícil [o início do trabalho de matriciamento] porque a lógica que tinha, que os PSFs tinham era de encaminhamento. (...) A forma de..., que entendiam é que se tivesse encaminhando tava tudo resolvido" (gerente CAPS).

\section{- Encaminhar como compartilhar}

Nos casos em que o termo encaminhar é empregado como compartilhar ou pedir de ajuda para situações específicas, os encaminhamentos são precedidos de uma discussão de caso entre profissionais das equipes da ESF e CAPSI. Tal discus- são varia de uma unidade para outra, ocorrendo por contato telefônico ou pessoalmente.

Havendo discussão prévia, ambas as equipes compartilham informações e saberes e decidem como lidar com a situação conjuntamente. Além disso, evita-se que o paciente vá de um serviço a outro em busca de cuidados que podem ou não ser acolhidos.

Entendemos que esse é o que tem sido denominado encaminhamento implicado e preconizado pelo Ministério da Saúde 2 (p. 13) como um princípio para os cuidados em saúde mental de crianças e adolescentes, que "exige que aquele que encaminha se inclua no encaminhamento, que se responsabilize pelo estabelecimento de um endereço para a demanda, acompanhe o caso até seu novo destino".

Salientamos que, para tornar o encaminhamento possível, é necessário que haja uma organização nos serviços que viabilize o contato entre as equipes e o acesso ao serviço CAPS, fatores que, quando presentes, são apontados pelos entrevistados como facilitadores da articulação. A existência de um canal aberto para essa discussão se mostra fundamental para seu funcionamento.

\section{Matriciamento - a palavra da vez}

O termo matriciamento ou apoio matricial apareceu nas falas de todos os sujeitos da pesquisa, embora não estivesse presente na entrevista. $\mathrm{Na}$ maioria dos casos, seu uso se referia à forma como, atualmente, as questões de saúde mental têm sido tratadas na ESF.

A rigor, o matriciamento refere-se a um arranjo organizacional horizontalizado que visa outorgar suporte técnico em áreas específicas às equipes responsáveis pela saúde integral de determinada população, denominadas dentro desse modelo como equipes de referência ${ }^{21}$. A equipe de apoio matricial, composta por especialistas em determinada área, atua de maneira complementar com as equipes de referência, como as da ESF. Com isso, amplia-se a oferta de ações em saúde usando saberes e práticas especializados sem que o usuário deixe de ser cuidado pela equipe de referência 21,22 , uma vez que ambas se corresponsabilizam pelos casos e compartilham a elaboração e execução dos projetos terapêuticos.

Em diferentes momentos históricos, três dos cinco CAPSI pesquisados já foram apoiadores matriciais de equipes de ESF, porém apenas um deles preservou essa função e a realiza conjuntamente com o CAPS adulto, CAPS álcool e drogas e Núcleo de Apoio à Saúde da Família (NASF). Todas as equipes de ESF incluídas nessa investi- 
gação recebem apoio matricial, e algumas passaram a tê-lo apenas a partir da inserção dos NASF, em 2008.

As falas analisadas indicam que, no trabalho prático entre apoiadores matriciais e equipes de referência, a corresponsabilização, principal característica desse arranjo 21 , nem sempre está presente. Assim, os entrevistados relatam que as equipes de apoio matricial têm, em algumas situações, operado para receber casos, realizar intervenções e indicar condutas, sem que haja um compartilhamento de saberes e de decisões entre os distintos profissionais. Dessa forma, a lógica centrada no encaminhamento ao especialista se mostra sobreposta à do matriciamento, o que vai de encontro à pesquisa realizada em Campinas 13 .

Na operacionalização do trabalho entre equipes de referência e apoio matricial, a análise do material coletado evidencia que não há consenso sobre as categorias de trabalhadores que devem se envolver no processo de matriciamento.

Como exemplo disso, temos a participação dos agentes comunitários de saúde (ACS) nas reuniões entre as equipes de referência e saúde mental. Enquanto alguns entrevistados pensam que sua presença é indispensável, outros indicam que sua participação pode ser pontual ou opcional. Lancetti 23 estima serem os ACS peças fundamentais nessa estratégia. Têm grande importância na articulação com a comunidade, pois têm poder de entrada e vínculo, podem contribuir para a elaboração e execução de projetos terapêuticos pautados na cidadania, no local de adoecimento e sofrimento das pessoas, na busca de novos caminhos, descentralizando o foco na doença no saber médico e na instituição10.

A falta de consenso ou fragmentação se faz, igualmente, presente no nível das ações de matriciamento. O contato entre as equipes pode acontecer por meio de: (1) encontros periódicos para discussão de casos, elaboração de projetos terapêuticos e intervenções; e (2) acionamento da equipe de apoio matricial em casos de maior urgência $^{19}$. Por mais que todas as unidades pesquisadas adotem a primeira forma como principal estratégia do apoio matricial, a segunda está presente apenas em alguns locais.

"Nós temos isso funcionando com a saúde mental em matriciamento. Então, o representante do CAPS infantil, ele tá vindo quinzenalmente junto com outro CAPS, que participa CAPS adulto, CAPS álcool e drogas, né (...). E sempre que precisa nós podemos fazer fora dessa..., do matriciamento, dependendo da urgência, via telefone, pode fazer contato" (gerente ESF).

Vale destacar que observamos na fala acima que o contato via telefone, para casos mais ur- gentes, está presente na articulação entre CAPS e ESF, mas é visto pelo entrevistado como uma estratégia que está "fora do matriciamento", o que conota o entendimento de matriciamento como sinônimo de reunião.

São mencionados, com frequência, problemas da rede de saúde pública, tais como escassez de recursos humanos e de serviços, rigidez nas agendas e cobrança por produtividade que atrapalham ou, até mesmo, inviabilizam o funcionamento do apoio matricial, pois não há espaço para discussão de casos, intervenções conjuntas e participação em cursos de capacitação.

“(...) eles [os médicos] têm uma agenda apertada, e mesmo eu pra dispensar outro profissional pra ir prum curso de matriciamento (...) a agenda tem que ser fechada, né. (...) Quando aparece um curso, eu tenho que pegar aquele bendito daquele dia, telefonar pros pacientes ou então mandar o agente comunitário nas casas avisando que não vai ter atendimento e arrumar um espaço nos outros dias pra pegar aqueles pacientes e colocar nas agendas" (gerente ESF).

Tais obstáculos impedem a realização de um trabalho compartilhado entre equipes e, consequentemente, um cuidado integral para a população.

\section{Parceria para casos de CAPSI}

Chamam a atenção os constantes impasses relatados nas entrevistas sobre o que é "caso de CAPS” que podem levar os usuários a peregrinarem à procura de atendimento e terminarem, muitas vezes, sem assistência. O questionamento que se faz é, sobretudo, acerca do que cabe a cada equipamento, havendo pouca menção sobre o trabalho em rede e a corresponsabilização pelos casos, o que indica um isolamento dos serviços em si mesmos e uma desarticulação de suas ações.

Conforme Portaria no. 336/02, os CAPS têm a função de ordenador de toda a demanda de saúde mental de sua área de abrangência. Contudo, podemos perceber que gerenciar todas as estratégias de saúde mental de uma região é uma tentativa que não se efetiva na prática. Ademais, o papel central do CAPS na rede de cuidados em saúde mental é questionável, pois pode corroborar para seu isolamento em relação aos serviços 13. Pensamos que as ações são mais eficazes se operadas em conjunto, utilizando diversos recursos comunitários com destaque para um ou outro equipamento dependendo do momento e singularidade do caso.

Cabe acrescentar que, sendo a atenção básica porta de entrada do sistema de saúde, muitos casos de saúde mental não chegam aos CAPS e po- 
dem, com sucesso, ser acolhidos e cuidados nesses locais. Se as equipes da ESF se voltam para a atenção integral da população e os CAPS cuidam de pessoas em intenso sofrimento psíquico, há uma intersecção entre ambas em situações que envolvem saúde mental, pois tratam das mesmas pessoas e fazem parte do mesmo SUS 24.

No entanto, as falas indicam que há uma tendência das unidades de passar a demanda para o outro, no caso das equipes da ESF, ou de "devolvê-la", no caso dos CAPS, muitas vezes por não saber como lidar com a situação ou por achar que aquilo não lhes cabe, o que acaba por gerar desassistência ao usuário.

“(...) a gente não conseguia lidar na rede não. O paciente ia procurar o serviço e simplesmente mandavam que não era caso pra lá e mandava de volta pra cá. (...) a gente indicava o CAPS e o contato era muito ruim, não consegue entrar (...) eles falam que não é caso dele. (...) A gente fica meio sem saber, ela surtava, aí vai, foram umas duas vezes que a Dra. pediu, escreveu, falaram que não era caso pra eles, retorna o paciente pra cá. Aí você fica, ai, e agora? O que que eu faço, né?" (gerente ESF).

Essa seleção (inclusão/exclusão) de usuários assumidos por cada serviço é criticada por Rotelli et al. 25 que afirmam que ela advém do elevado nível de especialidades e refinamento das técnicas interventivas que levam os serviços a funcionar segundo uma lógica de empresa: selecionam os problemas com base na própria competência e quanto ao restante podem dizer "não é um problema nosso". Isso "faz com que as pessoas sejam separadas, 'despejadas', jogadas de um lado para o outro entre competências diferentes e definitivamente não sejam responsabilidade de ninguém e sim abandonadas a si mesmas" 25 (p. 22). Nessas situações, “a 'estática' da segregação em uma instituição separada e total, o hospital psiquiátrico, é substituída pela 'dinâmica' da circulação entre os serviços especializados com prestações pontuais e fragmentadas" 25 (p. 23).

O critério comumente utilizado pelas equipes, de acordo com análise do material, para saber o que é "caso de CAPS" tem a ver com a gravidade do quadro ou com um momento de crise pelo qual o usuário esteja passando. Esse critério cria muitas dúvidas entre as equipes e vemos que há uma dificuldade de se estabelecer até que ponto o usuário pode ser atendido pela Atenção Básica e quando ele deve ir para o CAPS. Além disso, os entrevistados relatam que os casos "intermediários", ou seja, aqueles considerados nem tão leves nem tão graves, não tem local definido de tratamento.

"Os casos de crise que vão pro CAPS, né. Aí por isso a gente discute, porque às vezes a gente enten- de que é um caso de CAPS e às vezes não é. Ou às vezes a gente quer mandar um paciente pra CAPS que é um paciente pra Pronto-Socorro, né, que vai na verdade precisar passar a noite. Ele não vai resolver só com o dia" (gerente ESF).

O entendimento de que o CAPS tem o papel de atender o usuário apenas em uma situação de crise e depois "devolvê-lo para o território" para reinseri-lo socialmente nos remete ao modelo manicomial que opera o atendimento dentro de um local específico para depois devolvê-lo à sociedade. Sabemos que a intervenção terapêutica no contexto social das pessoas tem mais efetividade e, sendo assim, a articulação entre serviços se mostra necessária.

Levando em conta que o CAPS é um serviço de base territorial e comunitária, suas ações devem sair de dentro dos serviços e buscar vínculos que possam complementar seus recursos, evitando-se uma nova modalidade de cronificação, dentro dos CAPS 26. As filas de espera existentes em algumas unidades pesquisadas refletem seu isolamento em relação aos demais recursos da rede de saúde.

Chama a atenção o fato de as especificidades da infância e adolescência terem sido pouco mencionadas nas entrevistas realizadas. Na maioria dos casos, ainda que as perguntas versassem sobre esse público, os entrevistados, em especial da ESF, falavam dos CAPS de uma maneira geral e das dificuldades em lidar com saúde mental.

Pensamos que tal fato esteja relacionado com o próprio percurso histórico no qual a saúde mental se constitui no país, com foco na população adulta 1,27 , tendo a infância e adolescência sido delegada aos setores da Educação e Assistência Social 27. Apenas no início do século XXI foram elaboradas propostas para o desenvolvimento de uma política específica para crianças que estiveram até então "ausentes da agenda de debates, excluídas das proposições de políticas públicas de saúde mental, silenciadas nos documentos oficiais" 28 (p. 133).

Para Couto \& Delgado 29 , é o setor saúde que deve iniciar o processo de montagem da rede de cuidados, para que, em um segundo momento, seja possível que todos se envolvam igualmente e se impliquem em um problema "verdadeiramente comum" 29 (p. 279). Tal iniciativa demandaria, porém, uma aguda consciência prévia da importância e da especificidade representadas pelas crianças e adolescentes em sofrimento psíquico 30 .

A despeito do protagonismo da saúde mental, claro está que a intersetorialidade das ações se impõe como única possibilidade para construção de uma rede de serviços capaz de respon- 
der a complexidade dos cuidados a essa população ${ }^{29}$, posto que a condição de criança/adolescente, por si mesma, aciona vários setores, como a educação, a justiça, o lazer, entre outros ${ }^{31}$. A escola e as equipes de ESF são importantes dispositivos na construção de uma rede ampliada e inclusiva em saúde mental 27.

Acrescenta-se a isso o fato de que um olhar voltado para a saúde mental infantojuvenil nesses locais pode contribuir na questão da prevenção, detecção e intervenção precoces 27,31,32.

Por fim, vale dizer que as famílias têm importante papel nesse processo. As equipes da ESF, por lidarem diretamente com elas podem ter relevância nas ações de saúde mental se estiverem capacitadas e receberem apoio de outros profissionais. Assim sendo, o investimento na saúde mental infantojuvenil, além de beneficiar a própria criança, repercute na saúde mental da família como um todo 31 .

\section{Considerações finais}

A cidade de São Paulo representa um campo de pesquisa complexo e heterogêneo, dada a sua magnitude populacional e territorial, a grande variabilidade de condições socioeconômicas e culturais de seus habitantes, entre outros fatores.

A articulação das ações voltadas à saúde mental infantojuvenil realizadas pelos CAPSI e ESF ocorre de formas distintas nos locais pesquisados do município. Todavia, vemos em comum que a possibilidade de se incluir a saúde mental no escopo das ações das equipes de ESF vem se ampliando.

Percebemos que algumas equipes conseguem pôr em prática um modelo de saúde mental compatível com o modo psicossocial. Nesses casos, há grande empenho das equipes em trabalhar em rede, realizar parcerias com os recursos na comunidade, mesmo quando escassos, construir relações horizontais entre trabalhadores por meio do diálogo, fatores indispensáveis à concretização de um cuidado integral. No entanto, a lógica do encaminhamento, na qual se envia o usuário para serviços especializados e se transfere a responsabilidade por seu cuidado, é prática predominante. Esse modo de operar advém de um modelo tradicional de saúde que enxerga o sujeito de maneira fragmentada e desenvolve tecnologias voltadas ao indivíduo fracionado.

Para contrapor tal tendência, o matriciamento concebido como um arranjo organizacional capaz de dar sustentação aos equipamentos da rede de forma a operar de maneira integrada e articulada, possibilitaria a apreensão do sujeito em sofrimento em sua totalidade. Contudo, tal como observamos em alguns casos, fala-se em apoio matricial sem se compreender e operar o que nos parece fundamental de sua proposta: a corresponsabilização pelos usuários e a construção de ações conjuntas. Vale lembrar que tal arranjo é complementar ao de equipes de referência, que se responsabilizam longitudinalmente pela saúde integral de uma população. Apesar da incompatibilidade entre os princípios que regem a organização do apoio matricial e a lógica do encaminhamento, ambas têm se confundido na prática do trabalho e, por vezes, se mostram sobrepostas.

Não se pode, porém, perder de vista os obstáculos estruturais e organizacionais presentes no cotidiano dos serviços, tais como escassez de recursos humanos e de equipamentos de saúde, cobrança por produção, rotatividade de profissionais e falta de capacitação ou disposição para lidar com saúde mental. Semelhantes problemas precisam de ser devidamente abordados, embora não sejam determinantes para a superação do modelo tradicional.

Pensamos que, para além dos já conhecidos cursos de capacitação em saúde mental, o trabalho conjunto entre equipes de saúde mental e saúde da família, discutindo casos, compartilhando conhecimento, elaborando estratégias, pensando coletivamente em caminhos, realizando visitas domiciliares conjuntas ajudam os profissionais a se sentirem mais seguros e a perceberem que não estão sozinhos para "resolver" os casos. Podem, consequentemente, compartilhar responsabilidades e se fortalecer para lidar melhor com situações complexas, sem almejarem a rápida solução ou a cura daquele usuário, mas a ampliação de suas possibilidades de vida.

Assim, vimos que nos locais onde as equipes são mais próximas, os profissionais se conhecem, estabelecem laços pessoais e criam canais para contato sempre que necessário, lidar com saúde mental se torna menos problemático. Ainda que uma rede de atenção integral se componha pelos serviços de saúde e recursos do território, ela depende (e se constrói com) das pessoas que atuam nos serviços, das conexões e vínculos que estabelecem entre si para atingir um objetivo comum. O compartilhamento de responsabilidades e ações interventivas entre CAPSI e ESF só se realiza por meio das relações sociais entre os trabalhadores dos serviços. Portanto, valorizar e investir na relação entre as pessoas nos parece fundamental para que haja um trabalho compartilhado entre as duas equipes e na rede como um todo.

Sendo assim, o trabalho conjunto e articulado entre profissionais de CAPSI e ESF se mostra indispensável para garantir um cuidado integral voltado às crianças e aos adolescentes em sofri- 
mento psíquico. No entanto, sua concretização tem sido um desafio que se coloca no cotidiano de trabalho. Como vimos, o caminho é longo, os obstáculos são frequentes e os desafios constan- tes. Compete a todos os atores, usuários, trabalhadores, pesquisadores persistir na jornada para que possamos construir um sistema de saúde cada vez mais integral e respeitoso.

\section{Resumo}

O objetivo do estudo é descrever e analisar as articulações que se realizam entre as equipes da Estratégia Saúde da Família (ESF) e Centro de Atenção Psicossocial Infantojuvenil (CAPSI), tendo em vista as ações voltadas à saúde mental de crianças e adolescentes. Foram realizadas entrevistas semidirigidas com gerentes de cinco CAPSI e 13 Unidades Básicas de Saúde com ESF, de 5 regiões distintas no Município de São Paulo, Brasil, que foram transcritas e analisadas mediante perspectiva hermenêutica. A articulação entre as equipes da ESF e CAPSI se dá prioritariamente por encaminhamento de casos, apoio matricial ou parceria para casos considerados pertinentes ao CAPSI. Falta de recursos humanos, cobrança por produtividade e ausência de capacitação dos profissionais da ESF para trabalhar com saúde mental foram mencionadas como obstáculos para a efetiva articulação entre os serviços. A lógica do encaminhamento e da desresponsabilização, bem como a hegemonia do modelo biomédico e a consequente fragmentação dos cuidados se mostram vigentes no cotidiano dos serviços.

Saúde Mental; Serviços de Saúde Mental; Criança; Adolescente

\section{Colaboradores}

P. S. S. Delfini participou da coleta de dados, sistematização e análise dos dados e elaboração do artigo. A. O. A. Reis orientou a pesquisa, participou da sistematização, análise dos dados e elaboração do artigo. 


\section{Referências}

1. Reis AOA, Delfini PSS, Dombi-Barbosa C, Bertolino Neto MM. Breve história da saúde mental infantojuvenil. In: Lauridsen-Ribeiro E, Tanaka OY, organizadores. Atenção em saúde mental para crianças e adolescentes no SUS. São Paulo: Editora Hucitec; 2010. p. 109-30.

2. Departamento de Ações Programáticas Estratégicas, Secretaria de Atenção à Saúde, Ministério da Saúde. Caminhos para uma política de saúde mental infantojuvenil. Brasília: Ministério da Saúde; 2005.

3. Hoffmann MCCL, Santos DN, Mota ELA. Caracterização dos usuários e dos serviços prestados por Centros de Atenção Psicossocial Infantojuvenil. Cad Saúde Pública 2008; 24:633-42.

4. Delfini PSS, Dombi-Barbosa CD, Fonseca FL, Tavares CM, Reis AOA. Perfil dos usuários de um Centro de Atenção Psicossocial Infantojuvenil da Grande São Paulo, Brasil. Rev Bras Crescimento Desenvolvimento Hum 2009; 19:226-36.

5. Reis AOA, Delfini PSS, Dombi-Barbosa C, Oliveira MFAPB. Crianças e adolescentes em sofrimento psíquico atendidos nos Centros de Atenção Psicossocial Infantojuvenis. In: Lauridsen-Ribeiro E, Tanaka OY, organizadores. Atenção em saúde mental para crianças e adolescentes no SUS. São Paulo: Editora Hucitec; 2010. p. 186-210.

6. Coordenação de Saúde Mental/Coordenação de Gestão da Atenção Básica, Ministério da Saúde. Saúde mental e atenção básica: o vínculo e o diálogo necessários. Brasília: Ministério da Saúde; 2003.

7. Departamento de Ações Programáticas Estratégicas, Secretaria de Atenção à Saúde, Ministério da Saúde. Saúde mental no SUS: os centros de atenção psicossocial. Brasília: Mistério da Saúde; 2004. (Série F Comunicação e Educação em Saúde).

8. Souza AC. Ampliando o campo da atenção psicossocial: a articulação dos Centros de Atenção Psicossocial com a saúde da família. Esc Anna Nery Rev Enferm 2006; 10:703-10.

9. Nunes M, Jucá VJ, Valentin CPB. Ações de saúde mental no Programa Saúde da Família: confluências e dissonâncias das práticas com os princípios das reformas psiquiátrica e sanitária. Cad Saúde Pública. 2007; 23:2375-84.

10. Delfini PSS, Sato MT, Antoneli PD, Guimarães PO. Parceria entre CAPS e PSF: o desafio da construção de um novo saber. Ciênc Saúde Coletiva 2009; 14:1483-92.

11. Munari DB, Melo TS, Rocha BS, Soares CB, Medeiros M. Saúde Mental no contexto da atenção básica: potencialidades, limitações, desafios do Programa de Saúde da Família Rev Eletrônica Enferm 2008; 10:784-95. http://www.fen.ufg.br/revista/ v10/n3/v10n3a24.htm.

12. Bezerra E, Dimenstein M. Os CAPS e o trabalho em rede: tecendo o apoio matricial na atenção básica. Psicol Ciênc Prof 2008; 28:632-45.

13. Figueiredo MD, Onocko-Campos R. Saúde Mental na Atenção Básica à saúde de Campinas, SP: uma rede ou um emaranhado? Ciênc Saúde Coletiva 2009; 14:129-38.
14. Silveira DP, Vieira ALS. Saúde mental e atenção básica em saúde: análise de uma experiência no nível local. Ciênc Saúde Coletiva 2009; 14:139-48.

15. Minayo MCS. O desafio do conhecimento: pesquisa qualitativa em saúde. São Paulo: Editora Hucitec; 2004.

16. Denzin NK, Lincoln YS. O planejamento da pesquisa qualitativa: teorias e abordagens. Porto Alegre: Editora Editora Artmed; 2006.

17. Bardin L. Análise de conteúdo. Lisboa: Edições 70; 1977.

18. Amarante P. Saúde mental e atenção psicossocial. Rio de Janeiro: Editora Fiocruz; 2007.

19. Campos GWS, Domitti AC. Apoio matricial e equipe de referência: uma metodologia para gestão do trabalho interdisciplinar em saúde. Cad Saúde Pública 2007; 23:399-407.

20. Lucchese R, Oliveira AG, Conciani ME, Marcon SR. Saúde mental no Programa Saúde da Família: caminhos e impasses de uma trajetória necessária. Cad Saúde Pública 2009; 25:2033-42.

21. Campos GWS. Equipes de referência e apoio especializado matricial: um ensaio sobre a reorganização do trabalho em saúde. Cienc Saúde Coletiva 1999; 4:393-403.

22. Núcleo Técnico da Política Nacional de Humanização, Secretaria-Executiva, Ministério da Saúde. HumanizaSUS: equipe de referência e apoio matricial. Brasília: Ministério de Saúde; 2004. (Série B Textos Básicos de Saúde).

23. Lancetti A. Saúde mental nas entranhas da metrópole. In: Jatene AD, Lancetti A, Mattos SAF, Cruz MLS, Rodrigues MSM, Rocha SM. Saúde loucura: saúde mental e saúde da família. São Paulo: Editora Hucitec; 2001. p. 11-52.

24. Lancetti A, Amarante P. Saúde mental e saúde coletiva. In: Campos GWS, Minayo MCS, Akerman M, Drumond Júnior M, Carvalho YM, organizadores. Tratado de saúde coletiva. São Paulo: Editora Hucitec; 2006. p. 615-34.

25. Rotelli F, Leonardis O, Mauri D. Desinstitucionalização, uma outra via. In: Nicácio F, organizador. Desinstitucionalização. São Paulo: Editora Hucitec; 2001. p. 17-59

26. Alverga A, Dimenstein M. A reforma psiquiátrica e os desafios na desinstitucionalização da loucura. Interface Comun Saúde Educ 2006; 10:299-316.

27. Couto MCV, Duarte CS, Delgado PGG. A saúde mental infantil na Saúde Pública brasileira: situação atual e desafios. Rev Bras Psiquiatr 2008; 30:390-8.

28. Couto MCV. Novos desafios à reforma psiquiátrica brasileira: necessidade da construção de uma política pública de saúde mental para crianças e adolescentes. In: Cadernos de Textos de Apoio da III Conferência Nacional de Saúde Mental. Brasília: Ministério da Saúde; 2001. p. 121-30.

29. Couto MCV, Delgado PGG. Intersetorialidade: uma exigência da clínica com crianças na atenção psicossocial. In: Lauridsen-Ribeiro E, Tanaka OY, organizadores. Atenção em saúde mental para crianças e adolescentes no SUS. São Paulo: Editora Hucitec; 2010. p. 271-9. 
30. Lauridsen-Ribeiro E, Tanaka OY. Organização de serviços no Sistema Único de Saúde para o cuidado de crianças e adolescentes com problemas de saúde mental. In: Lauridsen-Ribeiro E, Tanaka OY, organizadores. Atenção em saúde mental para crianças e adolescentes no SUS. São Paulo: Editora Hucitec; 2010. p.147-69.

31. Elia L, Amaral N, Armony NS, Pinto RF, Santos KWA. Impactos da política de saúde mental infantojuvenil na clínica do autismo praticada em rede territorial de cuidado no Rio de Janeiro. In: Lauridsen-Ribeiro E, Tanaka OY, organizadores. Atenção em saúde mental para crianças e adolescentes no SUS. São Paulo: Hucitec; 2010. p. 211-29.
32. Lauridsen-Ribeiro E, Tanaka OY. Problemas de saúde mental das crianças: abordagem na atenção básica. São Paulo: Annablume; 2005.

Recebido em 23/Fev/2011

Versão final reapresentada em 12/Out/2011

Aprovado em 20/Out/2011 